\title{
Estimativas de parâmetros genéticos para características de desempenho de suínos em fase de crescimento e terminação
}

[Genetic parameter estimates of performance traits in swine during the growing and finishing periods]

\author{
R.A. Torres Filho ${ }^{1,5}$, R.A. Torres ${ }^{2,5 *}$, P.S. Lopes $^{2,5}$, C.S. Pereira ${ }^{2}$, R.F. Euclydes ${ }^{2,5}$, \\ C.V. Araújo ${ }^{3}$, M.A. Silva $a^{4,5}$, FC. Breda ${ }^{1}$ \\ ${ }^{1}$ Estudante de Pós-graduação - Universidade Federal de Viçosa \\ ${ }^{2}$ Universidade Federal de Viçosa \\ Av. P.H. Holfs s/n \\ 36571-000 - Viçosa, MG \\ ${ }^{3}$ Universidade Federal Rural da Amazônia \\ ${ }^{4}$ Escola de Veterinária da Universidade Federal de Minas Gerais \\ ${ }^{5}$ Bolsista do CNPq
}

\begin{abstract}
RESUMO
Foram utilizados dados de conversão alimentar, espessura de toucinho corrigida para $100 \mathrm{~kg}$, idade para atingir $100 \mathrm{~kg}$ e ganho de peso médio diário, para estimar parâmetros genéticos e fenotípicos de características de desempenho de suínos da raça Large White. As características estudadas foram avaliadas por sexo, em razão do manejo diferenciado para machos e fêmeas. Variâncias e covariâncias genéticas e fenotípicas foram estimadas pelo método da máxima verossimilhança restrita, usando modelo que incluiu os efeitos genéticos direto, materno e comum de leitegada. As estimativas de herdabilidade do efeito genético direto variaram de 0,13 a 0,55 , indicando que, para a maioria das características, o uso da seleção direta deve ser eficiente. Os valores das estimativas de herdabilidade do efeito genético materno foram baixos $(0,02$ a 0,05$)$. As estimativas do efeito comum de leitegada variaram de baixas a médias $(0,05$ a 0,18$)$. As correlações genéticas entre as características indicaram que, quando o objetivo do programa de melhoramento é a melhoria de todas as características, deve-se fazer seleção direta para conversão alimentar, espessura de toucinho e idade para atingir 100kg de peso vivo, e indireta para ganho de peso médio diário por meio da seleção da idade para atingir $100 \mathrm{~kg}$.
\end{abstract}

Palavras-chave: suíno, conversão alimentar, correlação genética, herdabilidade, toucinho, ganho de peso

\begin{abstract}
Data on feed gain ratio, age at 100kg, backfat thickness adjusted for 100kg and average daily weight gain were used to estimate genetic and phenotypic parameter for performance traits of Large White breed. Analyses were carried out separately by sex as a result of different management systems. Variance, genetic and phenotypic covariances were estimated by Restricted Maximum Likelihood method, including in the model the direct genetic effects, maternal effects and common litter effects. Estimates of heritabilities for the direct genetic effects ranged from 0.13 to 0.55 , suggesting direct selection response for these traits. The estimates for the maternal effect were low and ranged from 0.02 to
\end{abstract}

Apoio financeiro: $\mathrm{CNPq}$

Recebido para publicação em 19 de abril de 2004

Recebido para publicação, após modificações, em 10 de dezembro de 2004

*Autor para correspondência (corresponding author)

E-mail: rtorres@ufv.br 
0.05. The genetic correlations between traits indicated that selection program to improve all traits should be based on direct selection to improve age at $100 \mathrm{~kg}$, feed gain ratio and backfat thickness. Genetic gains on average daily gain should be based on indirect selection for age at $100 \mathrm{~kg}$.

Keywords: swine, daily gain, backfat thickness, feed:gain ratio, genetic correlation, heritability

\section{INTRODUÇÃO}

Em programas de melhoramento genético de suínos, a escolha das características a serem avaliadas é um passo fundamental para se estabelecer estratégias na obtenção do ganho genético. O processo de avaliação deve levar em conta a importância econômica da característica, a variabilidade genética, a facilidade de mensuração e o impacto dessa característica na atividade econômica, bem como a associação existente entre as características mensuráveis.

Dada a necessidade de diminuir os custos fixos de produção, quanto menor o tempo necessário para se atingir o peso de abate, maior será a lucratividade da criação. Ganho de peso médio diário e idade quando se atinge o peso de abate são medidas importantes na avaliação do desempenho dos animais. Outra característica que deve receber atenção especial é a deposição de gordura, pois o animal é menos eficiente na deposição de gordura que na deposição de carne e há, também, a crescente exigência de carcaça magra. Assim, os programas de melhoramento necessitam avaliar características de qualidade de carcaça. A espessura de toucinho é um indicador da quantidade de gordura acumulada, podendo ser obtida no animal vivo em diferentes locais do corpo.

A herdabilidade da característica é uma das propriedades genéticas das populações, essencial para a determinação das diretrizes dos programas de melhoramento. Também é importante a associação genética entre as características, avaliada pela correlação genética. Se a correlação genética for favorável e positiva, a seleção para uma característica implica mudanças na outra, pois a unidade de seleção é o animal ou o genoma completo e não apenas um conjunto de genes que agem sobre a característica específica (Roso et al., 1995).

Para características de desempenho, existem vários trabalhos no Brasil (Lopes, 1994; Roso et al., 1995; Torres Júnior, 1996; Costa, 1999; Pita,
2000). Neles observa-se uma certa variação entre as estimativas dos parâmetros genéticos, atribuída a diferenças entre raças, populações da mesma raça, constituindo indício de que, para maximizar os ganhos obtidos em um programa de melhoramento, é necessário obter parâmetros para a população em questão.

O trabalho teve como objetivo estimar os componentes de variância e covariância, as herdabilidades para os efeitos genético direto e materno e as correlações genéticas de características de desempenho de suínos.

\section{MATERIAL E MÉTODOS}

Os dados utilizados são provenientes de uma linha, em desenvolvimento, da raça Large White, mantida pelo programa de melhoramento da empresa Sadia S.A., localizada no estado de Santa Catarina, relativos ao período de 1993 a 1999.

Os animais são todos de uma mesma granja, e os leitões são mantidos na recria até a $12^{\mathrm{a}}$ semana de idade. Nessa idade, os machos são submetidos ao teste de desempenho individual e as fêmeas, ao teste de desempenho em baias coletivas. Adota-se o regime alimentar à vontade para ambos os sexos. A espessura de toucinho é mensurada em todos os animais, ao final do teste.

Os machos foram avaliados para conversão alimentar (CAM), espessura de toucinho corrigida para $100 \mathrm{~kg}$ (ETM), idade para atingir $100 \mathrm{~kg}$ (IDAM) e ganho de peso médio diário, do início ao final do teste (GPDM). As fêmeas, para espessura de toucinho corrigida para $100 \mathrm{~kg}$ (ETF), idade para atingir 100kg (IDAF) e ganho de peso médio diário do nascimento ao final do teste (GPDF). Os dados referentes à espessura de toucinho foram ajustados ao peso vivo aos $100 \mathrm{~kg}$, utilizando-se fatores de correção da própria empresa.

As estimativas dos componentes de (co)variância 
e dos parâmetros genéticos foram obtidas pelo programa "multiple trait derivative-free restricted maximum likelihood" (MTDFREML) descrito por Boldman et al. (1995), que utilizaram a metodologia da máxima verossimilhança, restrita livre de derivadas (DFREML). O MTDFREML utiliza o algoritmo simplex para localizar o mínimo de $-2 \log _{\mathrm{e}} \mathrm{L} \quad(\mathrm{L}=$ função de verossimilhança), e os componentes de(co)variância, que minimizam a função $-2 \log _{\mathrm{e}}$ L, são estimativas de máxima verossimilhança.

De acordo com Torres Filho et al. (2004), o modelo mais indicado para as características de desempenho nessa população inclui o efeito genético direto do animal, o efeito materno e o efeito comum de leitegada.

Como efeitos fixos, foram utilizados os grupos contemporâneos, os quais foram formados pela combinação da semana do ano $\left(1=48^{\mathrm{a}}\right.$ à $8^{\mathrm{a}}$ semana; $2=9^{\text {a }}$ à $21^{\mathrm{a}}$ semana; $3=22^{\mathrm{a}}$ à $34^{\mathrm{a}}$ semana; e $4=35^{\text {a }}$ à $47^{\text {a }}$ semana) com o ano de nascimento do animal.

As estimativas de herdabilidade total $\left(\hat{\mathrm{h}}_{\mathrm{T}}^{2}\right)$, direta $\left(\hat{h}_{d}^{2}\right)$ e materna $\left(\hat{h}_{m}^{2}\right)$, da correlação entre os efeitos genéticos direto e materno $\left(\mathrm{r}_{\mathrm{dm}}\right)$ e genética entre as características, e a proporção atribuída aos efeitos comuns de leitegada $\left(\hat{c}^{2}\right)$ foram obtidas a partir dos componentes de (co)variância.

\section{RESULTADOS E DISCUSSÃO}

As estimativas de variâncias genética aditiva direta $\left(\hat{\sigma}_{d}^{2}\right)$ e materna $\left(\hat{\sigma}_{m}^{2}\right)$, de covariância entre os efeitos genéticos direto e materno $\left(\hat{\sigma}_{\mathrm{dm}}\right)$, de variância de efeitos comuns de leitegada $\left(\hat{\sigma}_{\mathrm{p}}^{2}\right)$, de variância residual $\left(\hat{\sigma}_{\mathrm{e}}^{2}\right)$ e de variância fenotípica $\left(\hat{\sigma}_{\mathrm{f}}^{2}\right)$, obtidas em análise envolvendo uma e duas características, são apresentadas na Tab. 1.

As estimativas de variâncias obtidas em análise bicaracterística foram semelhantes às obtidas em análise unicaracterística. Johnson et al. (2002) também encontraram valores similares das estimativas de parâmetros genéticos para peso aos 100 dias de idade em análise unicaracterística e em análise bicaracterística com outras características de desempenho. Assim, discutiram-se os resultados apenas das análises unicaracterística. Considerando que as estimativas de componentes de variância normalmente são influenciadas pela medida adotada, as discussões dos parâmetros genéticos foram realizadas com base nas herdabilidades, nas correlações e nos coeficientes $\mathrm{c}^{2}$.

Estimativas de herdabilidades direta, materna e total, das correlações entre os efeitos aditivos direto e materno, da proporção atribuída aos efeitos comuns de leitegada e respectivos errospadrão são apresentadas na Tab. 2.

O valor de $\hat{h}_{\mathrm{d}}^{2}$ da conversão alimentar $(0,21)$, menor que os de 0,34 e 0,40 encontrados por Torres Júnior (1996), maior que 0,15 obtido por Hermesch et al. (2002), e semelhante aos 0,22 e 0,25 encontrados por Ducos et al. (1993) e aos 0,18 e 0,27 por Crump et al. (1997), refletiu tanto os efeitos das diferenças genéticas quanto os das diferenças de meio ambiente sobre as características, além dos efeitos dos modelos utilizados.

A estimativa de efeito comum de leitegada para conversão alimentar $(0,11)$ foi maior que a de 0,04 obtida por Crump et al. (1997), de 0,06 e 0,09 por Torres Júnior (1996) e de 0,09 por Ducos et al. (1993) para a raça Large White, entretanto, foi menor que 0,12 obtido para a raça Landrace. Esse valor caracteriza a contribuição do ambiente para a variabilidade entre leitegadas.

Os valores das estimativas de herdabilidade da idade para atingir $100 \mathrm{~kg}(0,13$ e 0,20$)$ foram menores que os observados por Pita (2000), de 0,24 a 0,27 , e por Chen et al. (2002), 0,35, a 0,44 , para atingir $113,5 \mathrm{~kg}$.

As estimativas de herdabilidade materna da idade para atingir $100 \mathrm{~kg} \quad(0,01$ e 0,05$)$ foram semelhantes às encontradas por Chen et al. (2002), que variaram de 0,01 a 0,05. O valor de $\hat{\mathbf{c}}^{2}$ da idade para atingir $100 \mathrm{~kg}$ de 0,18 está abaixo da amplitude de 0,22 a 0,29 encontrada por Li e Kennedy (1994) e acima de 0,12 a 0,16 verificada por Chen et al. (2002). 


\section{Arq. Bras. Med. Vet. Zootec., v.57, supl. 2, p.237-244, 2005}

Tabela 1. Estimativas de variâncias genéticas aditivas direta $\left(\hat{\sigma}_{\mathrm{d}}^{2}\right)$ e materna $\left(\hat{\sigma}_{\mathrm{m}}^{2}\right)$, de covariância entre efeitos genéticos direto e materno $\left(\hat{\sigma}_{\mathrm{dm}}\right)$, de variância devido aos efeitos comuns de leitegada $\left(\hat{\sigma}_{\mathrm{p}}^{2}\right)$, de variância residual $\left(\hat{\sigma}_{\mathrm{e}}^{2}\right)$, de variância fenotípica $\left(\hat{\sigma}_{\mathrm{f}}^{2}\right)$ e de covariâncias diretas $\left(\hat{\sigma}_{\mathrm{d}_{1,2}}\right)$, efeitos comuns de leitegada $\left(\hat{\sigma}_{\mathrm{p}_{12}}\right)$, residuais $\left(\hat{\sigma}_{\mathrm{e}_{12}}\right)$ e fenotípicos $\left(\hat{\sigma}_{\mathrm{f}_{12}}\right)$ para as características avaliadas ${ }^{1,2}$

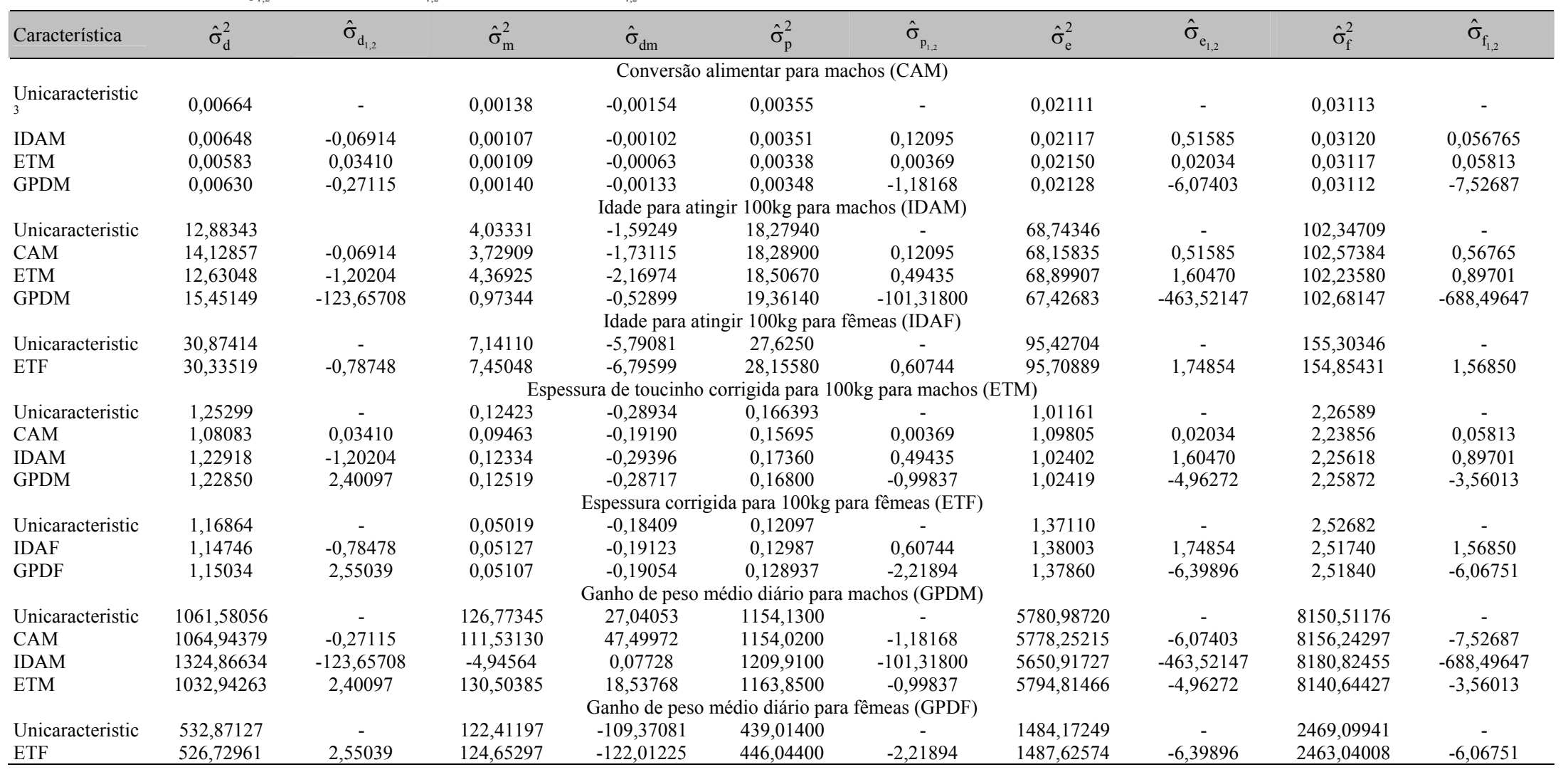

${ }^{1}$ Estimativas de variâncias obtidas da característica da linha, em análise bicaracterística, com a característica da coluna. ${ }^{2} \hat{\sigma}_{f_{12}}$ Os subitens 1 e 2 referem-se à característica da linha e a da coluna, respectivamente. ${ }^{3}$ Unicaracteristic $=$ Estimativas obtidas de análise de característica única. 
Tabela 2. Herdabilidades para efeitos genéticos aditivos direto $\left(\hat{\mathrm{h}}_{\mathrm{d}}^{2}\right)$ e materno $\left(\hat{\mathrm{h}}_{\mathrm{m}}^{2}\right)$, correlações entre efeitos aditivos direto e materno $\left(r_{\mathrm{dm}}\right)$, herdabilidade total $\left(\hat{\mathrm{h}}_{\mathrm{T}}^{2}\right)$, proporção atribuída aos efeitos comuns de leitegada $\left(\hat{c}^{2}\right)$ e estimativas dos respectivos erros-padrão (EP), obtidas da análise de característica única

\begin{tabular}{|c|c|c|c|c|c|}
\hline $\begin{array}{c}\text { Sexo e } \\
\text { característica }\end{array}$ & $\hat{\mathrm{h}}_{\mathrm{d}}^{2} \pm \mathrm{EP}$ & $\hat{\mathrm{h}}_{\mathrm{m}}^{2} \pm \mathrm{EP}$ & $r_{\mathrm{dm}} \pm \mathrm{EP}$ & $\hat{\mathrm{h}}_{\mathrm{T}}^{2}$ & $\hat{c}^{2} \pm \mathrm{EP}$ \\
\hline \multicolumn{6}{|c|}{ Conversão alimentar } \\
\hline Macho & $0,21 \pm 0,043$ & \multicolumn{3}{|c|}{ Idade para atingir $100 \mathrm{~kg}$} & $0,11 \pm 0,015$ \\
\hline Fêmea & $0,20 \pm 0,038$ & $0,05 \pm 0,018$ & $-0,39 \pm 0,310$ & 0,17 & $0,18 \pm 0,013$ \\
\hline Macho & $0,13 \pm 0,032$ & $0,04 \pm 0,020$ & $-0,22 \pm 0,357$ & 0,12 & $0,18 \pm 0,016$ \\
\hline \multicolumn{6}{|c|}{ Espessura de toucinho } \\
\hline Fêmea & $0,46 \pm 0,053$ & $0,02 \pm 0,013$ & $-0,76 \pm 0,500$ & 0,36 & $0,05 \pm 0,009$ \\
\hline Macho & $0,55 \pm 0,072$ & $\begin{array}{l}0,05 \pm 0,023 \\
\text { Ganho de pes }\end{array}$ & $\begin{array}{l}-0,73 \pm 0,380 \\
\text { édio diário }(\mathrm{g} / \mathrm{dia})\end{array}$ & 0,39 & $0,07 \pm 0,013$ \\
\hline Fêmea & $0,22 \pm 0,041$ & $0,05 \pm 0,019$ & $-0,43 \pm 0,311$ & 0,17 & $0,18 \pm 0,013$ \\
\hline Macho & $0,13 \pm 0,032$ & $0,02 \pm 016$ & $0,07 \pm 0,362$ & 0,14 & $0,14 \pm 0,015$ \\
\hline
\end{tabular}

As estimativas de herdabilidade direta da espessura de toucinho de 0,55 para machos e de 0,46 para fêmeas foram menores que a relatada por Lopes (1994), de 0,60, as de 0,63 e 0,65 relatadas por Johnson et al. (2002) e, em geral, maiores que as observadas por Torres Júnior (1996), 0,34 e 0,37, e por Costa (1999), 0,34 a 0,50. Crump et al. (1997) também encontraram valores diferentes para cada sexo, entretanto, ao contrário do observado neste trabalho, o valor para as fêmeas $(0,39)$ foi maior que para os machos $(0,30)$.

As estimativas de herdabilidade materna das características variaram de 0,02 a 0,05 e foram semelhantes às obtidas por Chen et al. (2002), que variaram de 0,02 a 0,04 , porém menores que as de 0,06 e 0,07 obtidas por Johnson (2002). Os valores de $\hat{\mathrm{c}}^{2}$ para espessura de toucinho corrigida para $100 \mathrm{~kg}$ para machos $(0,05$ e 0,07$)$ foram semelhantes aos encontrados por Costa (1999), de 0,05 a 0,09, porém menores que os encontrados por Chen et al. (2002), de 0,08 a 0,13 , e por Johnson (2002), de 0,08 a 0,10 .

$\mathrm{O}$ valor de $\hat{\mathrm{h}}_{\mathrm{d}}^{2}$ do ganho de peso médio diário para machos foi menor que os das fêmeas. Vale lembrar que o ganho de peso médio diário dos machos foi controlado a partir do início do teste (12 semanas de idade), em baias individuais, e o das fêmeas, desde o nascimento, em baias coletivas. Os valores de 0,13 para machos e de 0,22 para fêmeas foram menores do que os encontrados por Costa (1999), de 0,39 e 0,30, e de 0,32 e 0,33 por Pita (2000), e 0,28 e 0,26 por Johnson et al. (2002), porém semelhantes ao valor 0,15 observado por Hermesh et al. (2002).

As estimativas de herdabilidade materna foram de 0,02 e 0,05 para machos e fêmeas, respectivamente. Johnson (2002) também obteve estimativa de 0,02 tanto para um rebanho da raça Landrace como para Large White. Os valores de $\hat{\mathrm{c}}^{2}$ para ganho de peso médio diário $(0,14$ e 0,18$)$ foram semelhantes aos obtidos por Costa (1999), de 0,05 a 0,16 , e por Johnson et al. (2002), de 0,14 a 0,17 .

Pita (2000) e Torres Filho (2001), ao estudarem diversas características de desempenho de suínos, utilizando diferentes modelos, encontraram maiores estimativas de herdabilidade em modelos menos parametrizados. Assim, as magnitudes das estimativas obtidas neste estudo podem também ser atribuídas ao modelo utilizado na análise.

As estimativas de $\mathrm{c}^{2}$, que variaram de $0,05 \mathrm{a}$ 0,18 , evidenciam a importância da inclusão do efeito comum de leitegada nos modelos de avaliação das características de desempenho de suínos. Ducos et al. (1993), ao observarem que as estimativas do efeito comum de leitegada variaram de 5 a $16 \%$ da variância fenotípica para características de desempenho e carcaça, concluíram que esse efeito não poderia ser negligenciado na avaliação genética. As 
estimativas de $\mathrm{h}_{\mathrm{m}}^{2}$ das diferentes características foram relativamente baixas, semelhantes às observadas por Pita (2000), cujos valores de $\hat{\mathrm{h}}_{\mathrm{m}}^{2}$ das características de desempenho variaram entre 0,00 e 0,04. Porém, Torres Filho et al. (2004), ao avaliarem a inclusão de diferentes efeitos no modelo de avaliação genética de características de desempenho de suínos, encontraram evidências da importância da inclusão do efeito materno sobre essas características.

Com exceção do ganho de peso médio diário para machos, as estimativas de $r_{\mathrm{dm}}$ negativas, que variaram de $-0,22$ a $-0,76$, foram semelhantes às encontradas por Chen et al. (2002), de $-0,28$ a $-0,60$, por Johnson et al. (2002), de $-0,33$ a $-0,69$, e por Pita (2000), de $-0,26$ a $-0,45$.

Van Vleck et al. (1977), ao estudarem a resposta fenotípica esperada para gado de corte, com seleção para efeitos genético direto e materno, destacaram que se deve avaliar a magnitude da correlação genética entre esses dois efeitos, pois a magnitude dessa correlação pode prejudicar o ganho genético. Robinson (1996), ao examinar as correlações entre valores genéticos estimados por modelos alternativos, sugeriu que estimativas negativas e altas podem ser conseqüência da variação adicional entre reprodutores ou da interação reprodutor-ano. Pita (2000) relatou que as estimativas negativas e de média e alta magnitudes em características de desempenho de suínos podem estar relacionadas ao tamanho do conjunto de dados, à sua estrutura e à magnitude dos efeitos aditivos direto e materno.

As estimativas de correlações genéticas aditivas diretas entre características obtidas neste estudo encontram-se nas Tab. 3 e 4. Nas análises bicaracterísticas, que incluíram a idade para atingir $100 \mathrm{~kg}$ e o ganho de peso médio diário para fêmeas, não foi atingido o critério de convergência estipulado, provavelmente em razão de a IDAF ser o denominador da equação para obtenção do GPDF.

Tabela 3. Estimativas de correlações genéticas aditivas diretas, obtidas de características dos machos, em análise bicaracterística

\begin{tabular}{lcccc}
\hline \multirow{2}{*}{ Característica } & \multicolumn{3}{c}{ Correlação genética } \\
\cline { 2 - 5 } & CAM & IDAM & ETM & GPDM \\
\hline Conversão alimentar (CAM) & 1 & $-0,23$ & 0,43 & $-0,10$ \\
Idade para atingir 100 kg (dias) (IDAM) & & 1 & $-0,31$ & $-0,86$ \\
Espessura de toucinho (mm) (ETM) & & & 1 & 0,07 \\
Ganho de peso diário médio (gramas) (GPDM) & & & 1 \\
\hline
\end{tabular}

Tabela 4. Estimativas de correlações genéticas aditivas diretas, obtidas de características das fêmeas, em análise bicaracterística

\begin{tabular}{lcc}
\hline Característica & \multicolumn{2}{c}{ Correlação genética } \\
\cline { 2 - 3 } Idade para atingir 100 kg (dias) (IDAF) & IDAF & ETF \\
Espessura de toucinho (mm) (ETF) & 1 & $-0,13$ \\
Ganho de peso diário médio (gramas) (GPDF) & 1 & $-0,02$ \\
\hline
\end{tabular}

* A análise dessas duas características não atendeu aos critérios de convergência adotados.

A correlação obtida entre conversão alimentar e idade para atingir $100 \mathrm{~kg}$ indica relação desfavorável entre essas características, ou seja, os conjuntos gênicos que promovem aumento na idade no abate provocam aumento na conversão alimentar. Roso et al. (1995), Silva et al. (1992) e Torres Júnior (1996) também encontraram correlações negativas entre essas duas características, tendo o último autor destacado que, apesar da associação desfavorável, a sua baixa magnitude não inviabiliza a obtenção de progresso em ambas as características.

O valor positivo encontrado na associação entre conversão alimentar e espessura de toucinho corrigida para $100 \mathrm{~kg}$ é inferior aos valores 
apresentados por Silva et al. (1992) e Torres Júnior (1996), já a estimativa de 0,47 obtida por Hermesch et al. (2002), sob regime alimentar à vontade, foi próxima ao valor obtido neste estudo. Tendo em vista o interesse de se obterem animais com menores conversão alimentar e espessura de toucinho, a correlação genética positiva obtida é favorável à seleção. Apesar de a magnitude de 0,43 ser moderada, a seleção de apenas uma das características pode não ser recomendável, em razão da importância econômica das duas características, pois a resposta obtida da seleção indireta para conversão alimentar, conforme feito freqüentemente, seria menor do que a obtida pela seleção direta.

A associação genética negativa encontrada entre conversão alimentar e ganho de peso médio diário é favorável à seleção, entretanto, em razão de essa correlação apresentar baixa magnitude ($0,10)$, a resposta indireta de uma das características em função da seleção da outra não é recomendável. Hermesch et al. (2002) e Ducos et al. (1993) também encontraram valores negativos, porém de maiores magnitudes.

Um aspecto importante da conversão alimentar, que deve ser considerado, é o manejo da alimentação durante o teste de desempenho. Segundo Mcphee et al. (1988), no manejo com alimentação à vontade, as correlações entre ganho de peso diário e conversão alimentar são mais fracas em relação à obtida com a alimentação restrita.

A estimativa negativa da correlação genética entre idade para atingir $100 \mathrm{~kg}$ e espessura de toucinho corrigida condiz com os resultados obtidos por Li e Kennedy (1994), Silva et al. (1992), Torres Júnior (1996) e Chen et al. (2002). Essa associação é desfavorável à seleção, pois animais que apresentam menor idade aos $100 \mathrm{~kg}$ tendem a apresentar maior espessura de toucinho.

A alta e negativa correlação genética entre idade para atingir $100 \mathrm{~kg}$ e ganho de peso médio diário, em machos, era esperada, uma vez que o denominador do cálculo do GPDM é em função da idade do animal. Comportamento semelhante entre essas duas características foi também observado por Roso et al. (1995).
A correlação genética entre espessura de toucinho e ganho de peso médio diário foi negativa e de baixa magnitude, tanto para machos $(-0,10)$ quanto para fêmeas $(-0,13)$. Costa (1999) encontrou estimativas que variaram de 0,02 a 0,33. Hermesch et al. (2002) encontraram resultados divergentes dos obtidos neste trabalho, ou seja, valor negativo $(-0,18)$ sob regime alimentar restrito, e positivo $(0,12)$ sob regime alimentar à vontade. $\mathrm{O}$ sinal da associação encontrado entre essas duas características também não condiz com os argumentos de McPhee et al. (1988), ao relatarem que animais com crescimento mais rápido têm mais baixo conteúdo de carne na carcaça, quando alimentados à vontade. Li e Kennedy (1994) encontraram amplitude de $-0,06$ a $-0,17$, com média de $-0,13$, e ressaltaram que os sistemas de avaliação para espessura de toucinho e idade para atingir $100 \mathrm{~kg}$ são baseados em modelo animal unicaracterístico, e a seleção é realizada sobre um índice que combina as duas características, e que as baixas magnitude das estimativas da correlação genética entre duas características podem estar viesadas em razão da resposta à seleção.

\section{CONCLUSÕES}

As herdabilidades diretas estimadas para a maioria das características indicam a viabilidade de seleção direta. As herdabilidades maternas não indicam ganhos promissores por meio da seleção direta, havendo necessidade de melhor entendimento dos efeitos da estrutura dos dados e do número de informações sobre as estimativas das correlações direta e materna. As amplitudes das estimativas do efeito comum de leitegada evidenciaram a necessidade de sua inclusão em modelos de avaliação genética. As correlações genéticas entre as características de desempenho indicam que, em programas de melhoramento estabelecidos com objetivos de melhoria de todas as características, deve-se selecionar diretamente para conversão alimentar, espessura de toucinho e idade para atingir $100 \mathrm{~kg}$ e para ganho de peso médio diário pela seleção indireta na idade para atingir $100 \mathrm{~kg}$.

\section{AGRADECIMENTO}

À empresa SADIA S.A., pela concessão dos dados que possibilitaram a execução deste trabalho. 


\section{REFERÊNCIAS BIBLIOGRÁFICAS}

BOLDMAN, K.G.; KRIESE, L.A.; VAN VLECK, L.D. et al. A manual for use of MTDFREML: a set of programs to obtain estimates of variances and covariances [DRAFT]. Lincoln: U.S. Department of Agriculture/Agriculture Research Service,1995. $115 \mathrm{p}$.

CHEN, P.; BAAS, T.J.; MABRY, J.W. et al. Genetic parameters and trends for lean growth rate and its components in U.S. Yorksire, Duroc, Hampsire and Landrace pigs. J. Anim. Sci., v.80, p.2062-2070, 2002

COSTA, A.R.C. Avaliação genética e valores econômicos de características de desempenho em suínos. 1999. 50f. Dissertação (Mestrado) Departamento de Zootecnia, Universidade Federal de Viçosa, Viçosa, MG.

CRUMP, R.E.; HALEY, C.S.; THOMPSON, R. et al. Individual animal model estimates of genetic parameters for performance test traits of male and female Landrace pigs tested in a commercial nucleus herd. Anim. Sci., v.65, p.275-283, 1997.

DUCOS, A.; BIDANEL, J.P.; DUCROCQ, V. et al. Multivariate restricted maximum likelihood parameters for growth, carcass and meat quality traits in French Large White and French Landrace pigs. Genet. Sel. Evol., v.25, p.475-493, 1993.

HERMESCH, S.; LUXFORD, B.G.; GRASER, H.U. Feeding level influences genetic parameters for performance traits in pigs. In: WORLD CONGRESS ON GENETICS APPLIED TO LIVESTOCK PRODUCTION, 7., 2002, Monptellier, France. Proceedings... Montpellier, 2002. p.143-146.

JOHNSON, Z.B.; CHEWNING, J.J.; NUGENT III, R.A. Maternal effects on traits measured during postweaning performance test of swine from four breeds. J. Anim. Sci., v.80, p.1470-1477, 2002.

LI, X.; KENNEDY, B.W. Genetic parameters for growth rate and backfat in canadian Yorkshire, Landrace, Duroc and Hampshire pigs. J. Anim. Sci., v.72, p.1450-1454, 1994.

LOPES, P.S. Avaliação genética de suínos utilizando metodologia de modelos mistos. 1994. 98f. Tese (Doutorado) - Departamento de
Zootecnia, Universidade Federal de Viçosa, Viçosa, MG.

McPHEE, C.P.; RATHMELL, G.A., DANIELS, L.J. et al. Selection in pigs for increased lean growth rate on a timed feeding scale. Anim. Prod., v.47, p.149-156, 1988.

PITA, F.V.C. Modelos para avaliação genética e comparação de caracteríticas de desempenho para a seleção de suínos. 2000. 157f. Tese (Mestrado) Universidade Estadual Paulista, Jaboticabal, SP.

ROBINSON, D.L. Estimation and interpretation of direct and maternal genetic parameters for weights of Australian Angus cattle. Livest. Prod. Sci., v.45, p.1-11, 1996.

ROSO, V.M.; FRIES, L.A.; MARTINS, E.S. Parâmetros genéticos em características de desempenho e qualidade de carcaça em suínos da raça Duroc. Rev. Soc. Bras. Zootec., v.24, p.310316, 1995.

SILVA, M.A.; CATALAN, G.; TORRES, R.A. et al. Estimativas de componentes genéticos de características de importância econônica, em três diferentes raças de suínos. Rev. Soc. Bras. Zootec., v.25, p.923-932, 1992.

TORRES FILHO, R.A. Avaliação genética de características de desempenho e reprodutivas em suínos. 2001. 79f. Dissertação (Mestrado) Departamento de Genética e Melhoramento, Universidade Federal de Viçosa, Viçosa, MG.

TORRES FILHO, R.A.; TORRES, R.A.; LOPES, P.S. et al. Avaliação de modelos para estimação de componentes de (co)variância em características de desempenho e reprodutivas em suínos. Rev. Bras. Zootec., v.33, p.350-357, 2004.

TORRES JÚNIOR, R.A.A. Eficiência das informações de diferentes grupos contemporâneos na avaliação genética de suínos utilizando modelos mistos em procedimentos uni e multivariados. 1996. 117f. Dissertação (Mestrado). Departamento de Genética e Melhoramento, Universidade Federal de Viçosa, Viçosa, MG.

VAN VLECK, L.D.; LOUIS, D.S.; MILLER, J.I. Expected phenotypic response in weaning weight of beef calves from selection for direct and maternal genetic effects. J. Anim. Sci., v.44, p.360, 1977. 